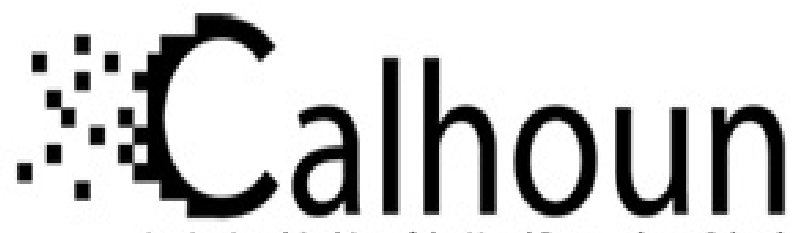

Institutional Archive of the Naval Postgraduate School

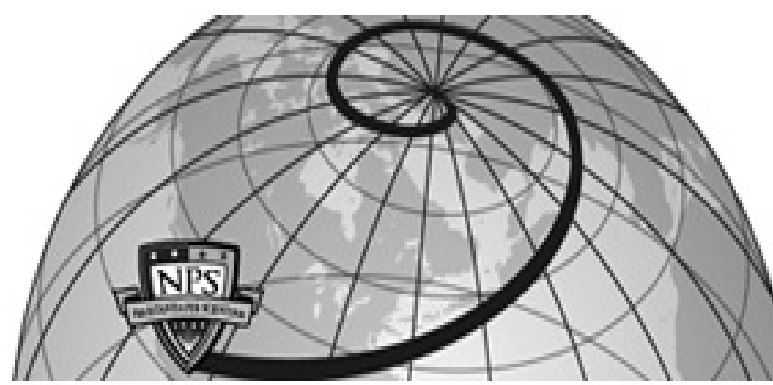

Calhoun: The NPS Institutional Archive

DSpace Repository

Bifocal Relay Mirror Experiments on the NPS

Three Axis Spacecraft Simulator

Spencer, M.; Chernesky, V.; Baker, J.; Romano, M.

AIAA-2002-5031, AIAA Guidance, Navigation and Control Conference, Monterey, CA, August 2002.

http://hdl.handle.net/10945/34489

This publication is a work of the U.S. Government as definedin Title 17, United States Code, Section 101. As such, it is in thepublic domain, and under the provisions of Title 17, United StatesCode, Section 105, is not copyrighted in the U.S.

Downloaded from NPS Archive: Calhoun

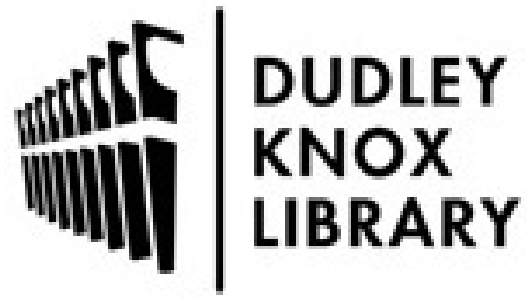

http://www.nps.edu/library
Calhoun is the Naval Postgraduate School's public access digital repository for research materials and institutional publications created by the NPS community. Calhoun is named for Professor of Mathematics Guy K. Calhoun, NPS's first appointed -- and published -- scholarly author.

Dudley Knox Library / Naval Postgraduate School 411 Dyer Road / 1 University Circle Monterey, California USA 93943 


\title{
BIFOCAL RELAY MIRROR EXPERIMENTS ON THE NPS THREE AXIS SPACECRAFT SIMULATOR
}

\author{
Michael G. Spencer*, Vince Chernesky ${ }^{\dagger}$, Jeffrey Baker ${ }^{\ddagger}$, Marcello Romano \\ Spacecraft Research and Design Center \\ Department of Aeronautics and Astronautics \\ Naval Postgraduate School \\ Monterey, CA 93943
}

\section{ABSTRACT}

The Three Axis Satellite Simulator (TASS) is a 4-foot diameter octagonal platform supported on a spherical air bearing. The platform hosts several satellite subsystems, including rate gyros, reaction wheels, thrusters, sun sensors, and an onboard control computer. This free-floating design allows for realistic emulation of satellite attitude dynamics in a laboratory environment. The Bifocal Relay Mirror spacecraft system is composed of two optically coupled telescopes used to redirect the laser light from ground-based, aircraft-based or spacecraft based lasers to distant points on the earth or in space for a variety of non-weapon, force enhancement missions. A developmental version of this system was integrated onto the TASS as an auxiliary payload. The objective of this research was to develop and test the integrated optics and TASS system. This effort included hardware design, fabrication, and installation; platform mass property determination; and the development and testing of control laws and signal processing routines utilizing MATLAB and SIMULINK. The combination of the TASS with the bifocal relay mirror payload allowed for dynamic, real-time testing and validation of the target acquisition, tracking, and laser beam pointing technologies as well as satellite stabilization.

\section{INTRODUCTION}

During the late 1980 s and early 1990 s interest in space-based mirrors was expressed for the purpose of furthering the Strategic Defense Initiative (SDI) program. Most notable of these experiments was the Relay Mirror Experiment (RME), which successfully proved the technology involved in targeting a ground-based laser on an orbiting satellite and

\footnotetext{
* Assistant Professor, Dept. of Aeronautics and Astronautics, Naval Postgraduate School, Member AIAA

$\dagger$ LT USN, Master of Science, Astronautical

Engineering

* Boeing Albuquerque, NM, Supporting AFRL/DE

- Ph.D., U.S. National Research Council Associate Fellow. AIAA Member.
}

successfully delivering reflected laser radiation to another ground facility ${ }^{1}$. The RME stemmed from the SDI requirement for a space platform capable of reflecting a beam from a cooperative ground based laser to another cooperative space-mirror. This spacecraft was the first step in meeting the challenges particular to this mission, including spacecraft and beam pointing and tracking, and spacecraft jitter control. The RME also demonstrated autonomous spacecraft attitude control, receiving only a telemetry update daily ${ }^{1}$.

Three lasers were used in the operation of the RME, two beacon lasers and the main relay beam. A beacon laser was originated at both source and target ground sites towards the RME spacecraft. These beams entered an onboard optical train that sensed the orientation of each incoming beam, and slewed the primary mirror to the proper angle to reflect the main beam from the source to the target. The reflected main relay beam and source beacon beam were sensed at the target location. Jitter and accuracy were measured both at the target site and onboard the spacecraft during each encounter. The tests were successful and the results were significantly better than expected, creating a new benchmark for future systems to be measured against.

The ultimate goals of this system were a space-based anti-ballistic missile system using mirrors to engage the target missiles. However, changes in public policy dictated that the SDIO (now Ballistic Missile Defense Organization (BMDO)) shift its focus away from the space mirror concept. The Air Force continued working the technical challenges of laser acquisition, tracking, and pointing, concentrating its efforts on the Airborne Laser (ABL) system. The ABL system has been highly successful, and much expertise has been gained in the area of jitter control, beam tracking and pointing, and beam forming.

In the late 1990s, a concept study performed by AFRL validated potential missions for a spacebased optical relay mirror for imaging and intelligence purposes, incorporating technologies developed in the decade since the RME. In 2000, a team of Naval Postgraduate School Masters Degree students completed a preliminary design of a satellite, 


\section{Bifocal Relay Mirror Spacecraft}

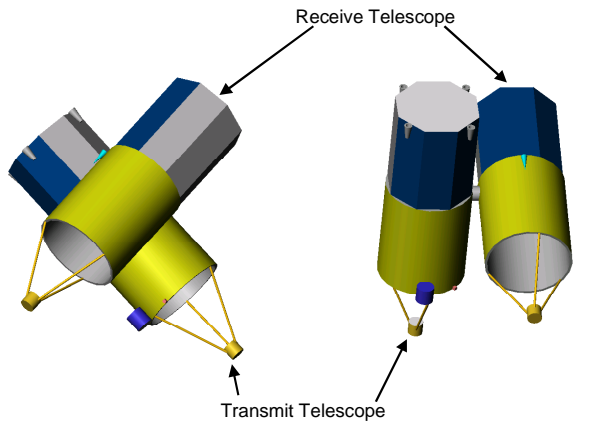

resulting in the scissors-like Bifocal Relay Mirror spacecraft $^{2}$ shown in Figure 1.

Figure 1. Bifocal Relay Mirror Spacecraft

The Bifocal Relay Mirror spacecraft consists of two optically coupled telescopes used to redirect the light from a ground-based laser to a distant target. A receiver telescope collects the incoming laser energy and channels it through internal relay optics to a transmitter telescope. The transmitter telescope directs the energy against the desired target. The relay optics between the two telescopes includes adaptive optics for correcting wave front aberration and beam steering mirrors ${ }^{2}$.

In December 2000, a proposal was submitted by NPS and AFRL to the National Reconnaissance Office under the Directors Innovation Initiative (DII) ${ }^{3}$. The DII program allocates funds to perform research efforts with significant payoff potential for space-based reconnaissance. This contract was awarded to NPS and AFRL in January 2001.

As part of this DII effort, a series of experimental tests were used to demonstrate and validate the integration of the Bifocal Relay Mirror concepts. These experiments provide a test bed to apply the latest technologies to the problems of beam control and tracking. This test bed incorporates spacecraft attitude dynamics and control, onboard jitter reduction and beam control while tracking an uncooperative target. This will prove the latest technologies in this area and provide experimental data useful in constructing a ground-based, multibody Bifocal Relay Mirror simulator.

\section{EXPERIMENTAL TEST PLAN}

The objectives of the experiments were to validate the acquisition, tracking and pointing control of the laser beam using steering mirrors integrated onto the NPS Three-axis spacecraft simulator (TASS). The TAS is a platform with reaction wheels, thrusters, rate gyros, sun sensor and control computer that can simulate the attitude dynamics of a spacecraft in orbit by floating on a spherical air bearing. AFRL provided fast steering mirrors, optics, sensors, lasers and beam control system while NPS provided the Three-axis spacecraft simulator as a movable spacecraft platform for the steering mirror and optics. The experiments were divided into phases:

Part A: Demonstrate beam jitter control with fast steering mirror on the fixed TASS

Part B: Track a moving target with cameras and fast steering mirror on the fixed TASS

Part C: Demonstrate jitter control and target tracking with the TASS platform floating and holding a fixed attitude.

In each experiment, the laser source was fixed and placed 1-2 meters from the TASS. During Parts A and B the TASS was held fixed and did not float. Part $\mathrm{C}$ experiments included the TASS floating with the laser source fixed off the platform.

Part A experiments were conducted at AFRL with the jitter control system on a typical optical bench prior to shipping to NPS and integrating onto the TASS. The tests successfully minimized the beam jitter caused by vibrating the source laser. Part B experiments involved the tracking of a green laser designator in a target screen with a camera system. A separate, off-board control computer processed the image and commanded the fast steering mirror to direct the red source laser to follow the target. During these tests the TASS was fixed and Figure 2 shows the hardware integrated onto the TASS for Part A \& B tests. These experiments successfully demonstrated the closed loop control of the source laser to track the green target spot.

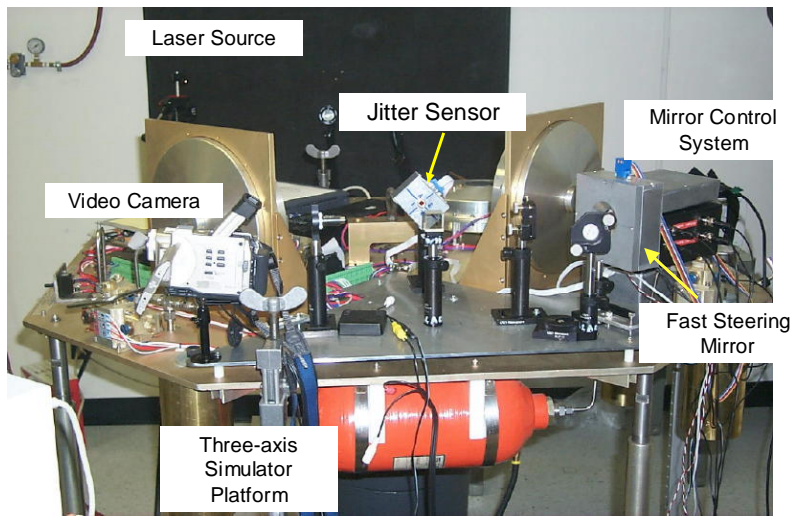

Figure 2. Target tracking hardware integrated onto the TASS. 
The following sections describe the TASS hardware and software as well as the integrated testing with the Bifocal Relay Mirror Payload (BRMP). This process comprised several simultaneous areas of research, experimentation, and development. Following the TASS delivery, the hardware/software interface required characterization, and the BRMP was integrated onto the TASS structure. The mass properties of the table required analysis and experimental validation, and calibration curves for the sun sensors were constructed. The power system required redesign to provide adequate capacity at several voltages, and the reaction wheel control system required a safety circuit to prevent damage. Several sensors proved to be noisy, requiring the development of signal processing algorithms to provide smooth data to the control laws. A PID controller was implemented, and direction cosine matrices were used to align the principal axes with the control axes. This development concluded with a successful test of the TASS and the BRMP.

\section{HARDWARE DEVELOPMENT}

The Three-Axis Satellite Simulator consists of several subsystems that act together to simulate satellite functions and attitude dynamics. This section outlines the major subsystems, its function, physical location, and operation.

The base structure is an octagonal aluminum plate, .375 " thick, supported by several aluminum, stiffening bars on the bottom side. A ten-inch diameter spherical air bearing is rigidly attached to the underside in the center of the plate. This air bearing sits in an air-bearing cup, which provides a smooth surface for the bearing to rest in when air is not applied to the cup. When air is applied to the cup, it raises the table $3 / 8$ " to a free-floating position.

The TASS also has four balancing legs, four ballast weights, and a three axis fine balance weight system on the underside. The balancing legs are adjustable up and down for changing the center of mass in the vertical direction. Small weight rings that fit around these legs provide the capability for gross balance adjustment. The ballast weights offset the large mass of equipment on the top surface of the table. The fine balancing weights allow for minute adjustment of the center of mass in all three axes.

Subsystems on the table include three orthogonally mounted reaction wheels, three orthogonally mounted rate gyros, a three-axis sun sensor, a three-axis magnetometer, a laptop computer, three lead-acid batteries, and a thruster system of two nitrogen propellant tanks and four thruster blocks providing three-axis control. Figure 3 outlines the locations of these components.

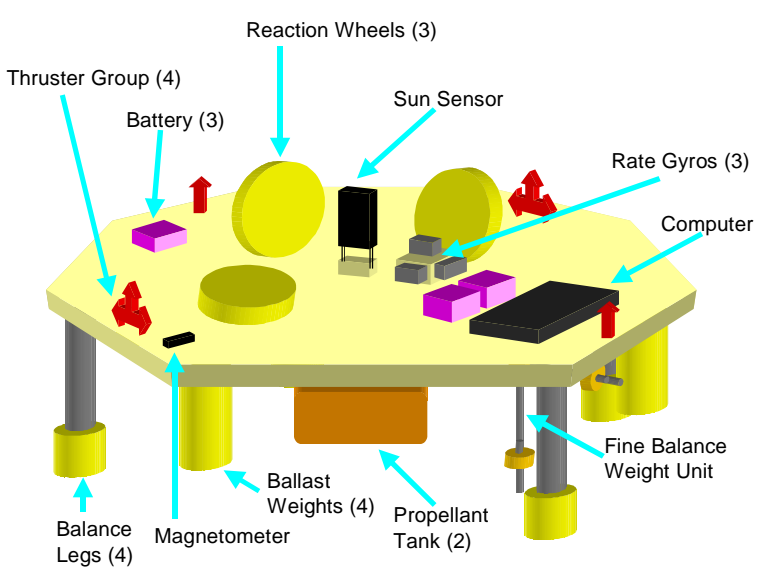

Figure 3. Subsystem Layout

The BRM payload consists of three electronics boxes for signal decoding, laser position determination, and fast steering mirror positioning. These boxes are mounted on an aluminum plate on the table surface above one of the propellant tanks. The optical train, which includes the fast steering mirror, is on a similar aluminum plate on the table surface above the other propulsion tank. A digital video camera is mounted alongside the optical train. The receiver, transmitter, and video power supply are located in available space along the edge of the table, Figure 4. Figure 5 is a photograph of the TASS from above.

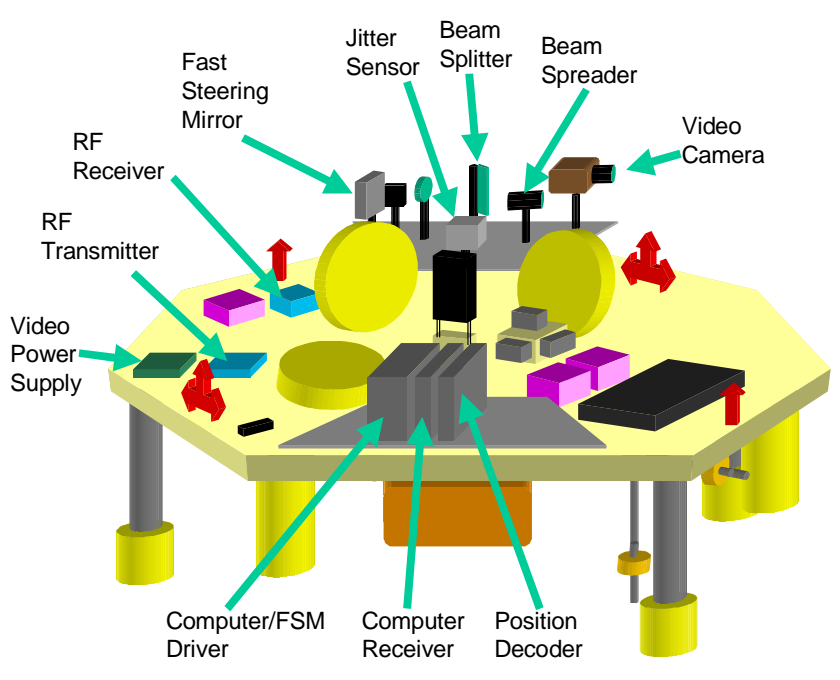

Figure 4. Bifocal Relay Mirror Payload Layout 


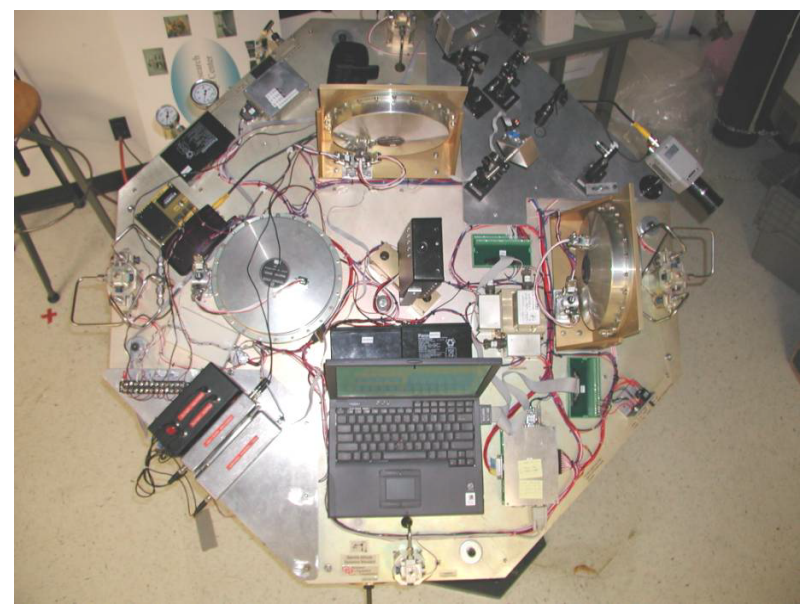

Figure 5. TASS Top View

\section{Reference Frames and Axes}

The TASS was delivered with a labeled, left-handed coordinate axis system (refered to as GDC) and a control program designed to operate around these axes. The operation of this program about these axes was demonstrated at time of delivery, however this demonstrated control was focused around thruster-based control, with a lightly damped demonstration of reaction wheel control. The BRMP requires fine attitude control, and it was decided that a reaction-wheel based control law would be implemented to meet the payload requirements. Not using the thrusters also eliminates the effect of mass loss due to propellant expulsion, and its subsequent effects on mass properties.

Based on the directions associated with positive sensor outputs, a right-handed control axis system was created and adopted as the standard system for all future table development (Figure 6). This axis system is referred to as the 'Control Axes', and a subscript of ' $\mathrm{C}$ ' denotes this axis $\left(\mathrm{X}_{\mathrm{C}}, \mathrm{Y}_{\mathrm{C}}, \mathrm{Z}_{\mathrm{C}}\right)$. The use of roll, pitch, and yaw are frequently associated with the attitude control of spacecraft and aircraft. For the purposes of the TASS it is useful to think of the user 'flying' the simulator from the location of the computer. Using this as a reference, roll is associated with motion about the $\mathrm{Z}_{\mathrm{C}}$ axis, pitch is associated with motion about the $X_{C}$ axis, and yaw is associated with motion about the $\mathrm{Y}_{\mathrm{C}}$ axis.

\section{Principal Axes and Moments of Inertia}

The TASS was originally constructed to have its principal axes coincident with the GDC axes. However the addition of ballasting weights, the bifocal relay mirror payload, and shifting the sun sensor location had the effect of changing the

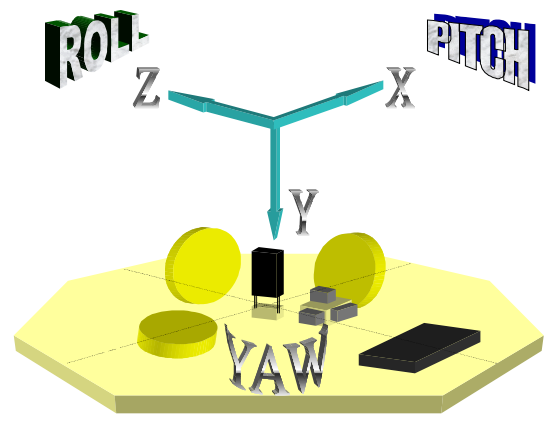

Figure 6. NPS Control Coordinate System

principal axes of the TASS. The moments of inertia were calculated for each component on the table, then each components MOI was translated into the mass properties frame via the parallel axis theorem. The principal moments of inertia of the TASS were calculated to be $I_{x x}=29.24 \mathrm{~kg}-\mathrm{m}^{2}, I_{y y}=29.07 \mathrm{~kg}-\mathrm{m}^{2}$, and $\mathrm{I}_{\mathrm{zz}}=45.01 \mathrm{~kg}-\mathrm{m}^{2}$.

In order to determine the actual principal axes, the TASS was first finely balanced, then one pound weights were fitted to the bottom of each balancing leg. This had the effect of lowering the center of mass of the table a fixed, calculable amount below the center of rotation. This known distance was coupled with the tables' pendulum period and mass to determine the moment of inertia.

An object can only oscillate about an axis without nutation if that axis is a principal axis. The pendulum testing was accomplished by floating the table and depressing one side until it reached the limit of the air bearing. The table was then smoothly released to impart no external force. The table then entered a pendulum-like state, with the air bearing and air friction on the table itself providing a small amount of damping. If nutation was observed after a short period of time, the table was clearly not oscillating about a principal axis. This process was repeated at intervals $\left(\sim 5^{\circ}\right)$ around the table, until no nutation was observed. Two axes were discovered to meet these criteria on the table approximately 90 degrees apart. This indicates that the $\mathrm{Y}_{\mathrm{C}}$ axis is coincident with the $Y_{P}$ axis, and that the control axes and principal axes are coupled by a single rotation about the Y-axis.

The periods of nutation about these two axes were $\mathrm{T}_{\mathrm{x}}=13.51$ seconds, and $\mathrm{T}_{\mathrm{z}}=13.16$ seconds. The mass added was 4 pounds, 15 inches below the center of rotation. The pendulum equation is: 


$$
T=2 \pi \sqrt{\frac{I}{m g \ell}}
$$

where $T$ is the period, $I$ is the moment of inertia, $m$ is the mass, $g$ is the acceleration due to gravity, and $\ell$ is the distance between the center of mass and center of rotation. Based on this data, the empirically derived moments of inertia about the $X_{P}$ and $Z_{P}$ axes were $I_{x x}$ $=31.3 \mathrm{~kg}-\mathrm{m}^{2}$ and $\mathrm{I}_{\mathrm{zz}}=29.7 \mathrm{~kg}-\mathrm{m}^{2}$. These are very close to the values of $29.2 \mathrm{~kg}-\mathrm{m}^{2}$ and $29.1 \mathrm{~kg}-\mathrm{m}^{2}$ analytically derived in the spreadsheet, validating the mass properties model.

\section{Rate gyros}

The TASS has three Humphrey rate gyros mounted on the top surface in a mutually orthogonal configuration. These rate gyros provide rate data to the TASS. The output signals of these rate gyros are noisy compared to the signal generated at the low angular rates experienced by the table during normal operation. Additionally the gyros have a significant bias, and this bias is not constant from day to day. These factors led to the implementation of two filters in order to provide accurate data.

\section{$\underline{\text { Sun sensors }}$}

The TASS initially had a two-axis sun sensor mounted to the left-hand side of the laptop computer on the platform. During subsequent testing, it was determined insufficient data existed to stabilize the TASS using only sun sensor and rate gyro data alone. The sun sensor was then removed and modified by to incorporate a third axis of information. The sun sensor was re-mounted in the center of the table facing the ceiling. A 12 foot blackout canopy was built 5 feet above the table, and two lights roughly one foot apart were placed in the center of this canopy to simulate the sun and a single star. The sun sensor used one of these lights to provide roll and pitch position information, and the position of the second light relative to the first light to provide yaw information.

The 'main' bulb is placed directly over the sun sensor, and is used to determine the pitch and roll of the table. The second bulb is placed along the wide dimension of the sun sensor, and determines the zero point of the yaw axis. The outputs of the sun sensor are a linear signal from -5 volts to +5 volts directly proportional to the angular displacement. Once this new sensor was installed, the slope and intercept of the linear equation describing angular position were determined in order to transform the voltage signal to an angular measurement in degrees.
For the $\mathrm{X}_{\mathrm{C}}$ and $\mathrm{Z}_{\mathrm{C}}$-axes this was accomplished by fixing a laser on the table perpendicular the axis to be measured. The table was floated and leveled using a bubble level, and the laser beam position was marked on the wall next to the TASS. This served as the 'zero' position for the axis. Measurements were marked on the wall in inches above and below this zero position (typically in 2 or 5 inch increments), and the distance from the center of rotation to the wall was measured. The table was then rotated so as to put the laser beam on each of the marks on the wall and the corresponding voltage recorded. Once this data was collected for the $\mathrm{X}$ and $\mathrm{Z}$ axes the angles to each mark on the wall were calculated using trigonometric equations, and the voltage data fit to the angular data using a short Matlab routine. A similar process was used to measure angular data for the Y-axis.

The data points at the limits in each axis represent the maximum achievable output in that axis, and are therefore indicative of the maximum field of view (FOV) in each axis. The $\mathrm{X}_{\mathrm{C}}$ axis has a FOV of $44^{\circ}\left(+25^{\circ}\right.$ to $\left.-19^{\circ}\right)$, the $\mathrm{Y}_{\mathrm{C}}$ of $90^{\circ},\left( \pm 45^{\circ}\right)$, and $\mathrm{Z}_{\mathrm{C}}$ of $9^{\circ},\left(+2^{\circ}\right.$ to $\left.-7^{\circ}\right)$. The $\mathrm{X}_{\mathrm{C}}$ and $\mathrm{Y}_{\mathrm{C}}$ axes are roughly centered on zero, while the $Z_{C}$ axis is significantly offset from the zero position. This is due to the physical positioning of the sun sensor on the table. Figure 7 shows the $\mathrm{X}_{\mathrm{C}}$ and $\mathrm{Z}_{\mathrm{C}}$ fields of view with respect to the sun sensor.

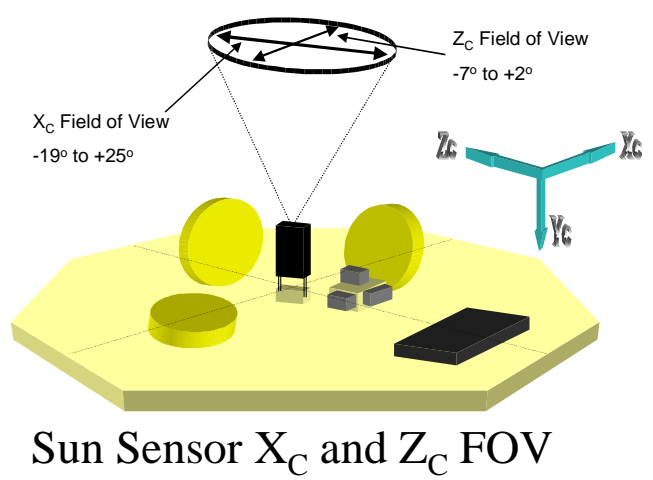

Figure 7. Sun Sensor Field of View

\section{Video system}

The BRMP was developed and tested independently in Albuquerque, New Mexico by Air Force Research Laboratory. This hardware consists of three electronics boxes for signal reception, beam position decoding, and the fast steering mirror (FSM) controller.

The operational concept of the BRMP is outlined in Figure 8. A red bench-top laser is aimed 
at the payload, which directs the laser at the FSM and then through a beam spreader to the target on a wall in the lab. Onboard the platform, the laser beam passes through a splitter, and high-frequency jitter is sensed and removed with a closed loop controller to the FSM. The video camera sends an image of the target to the control computer (located off the floating platform), which processes the image and sends fast steering mirror commands back to the floating platform to drive the red laser beam towards the green laser target.

The optical train consists of a primary mirror, the FSM, a lens, a variable beam splitter, a jitter sensor, and a beam spreader. A video camera is mounted adjacent to the optical train to provide feedback for beam targeting and steering. A diagram of the optical train is contained in Figure 9. The beam spreader is an inverted microscope lens, amplifying small motions of the FSM into larger motions on the target (A map of the world, hung on a wall ten feet away).

A radio frequency $(\mathrm{RF})$ transmitter/receiver transmits the video camera signals to a desktop computer for image processing, and another transmitter/receiver receives commands from the desktop computer to drive the FSM.

Integrating the BRMP onto the TASS presented some challenges. Besides the obvious changes in the mass properties of the table, providing power to the BRMP was a major issue. During the design process, it was thought that power could be brought to BRMP components by way of a lightweight umbilical. However, subsequent testing indicated that even the smallest disturbance in the mass characteristics of the table had a serious impact on its operation. This ruled out any sort of umbilical during table operation.

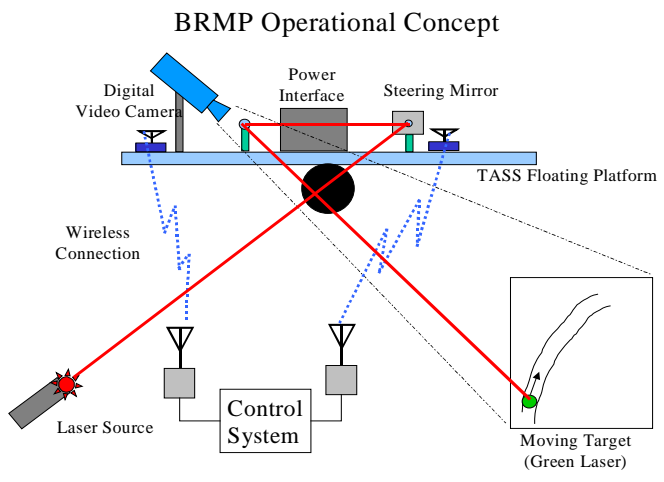

Figure 8. Operational Concept for Free-floating Tests

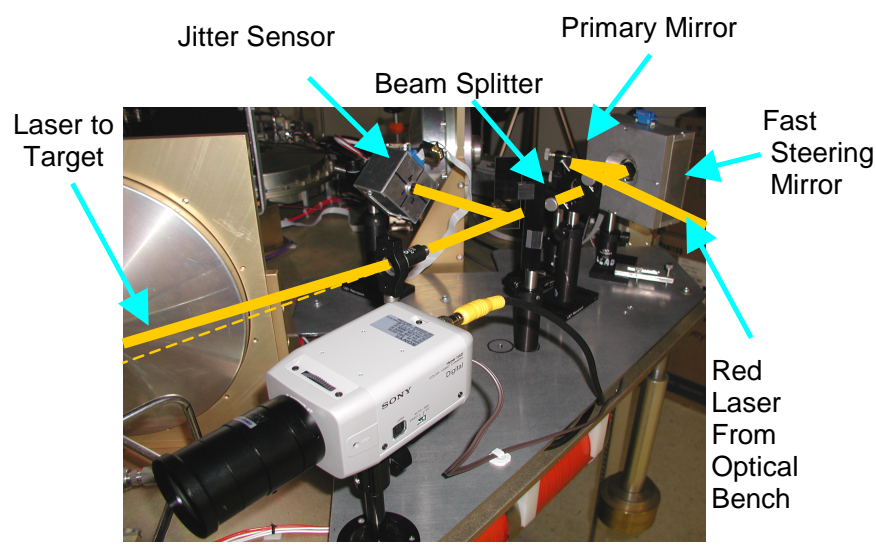

Figure 9. Optical Train Diagram

\section{SOFTWARE DEVELOPMENT \& SIGNAL $\underline{\text { PROCESSING }}$}

The Matlab/Simulink software package was used to interface with the National Instruments cards in order to process inputs from the table and provide appropriate command signals back to the table. This section reviews the software packages used, how the control laws were implemented and the signal processing required to make the input signals useable.

Sensor inputs to the computer and control outputs from the computer are via two National Instruments DAQcard-1200 PCMIA cards. Each card contains eight $\pm 5 \mathrm{~V}$ analog inputs, two $\pm 5 \mathrm{~V}$ analog outputs, and 24 digital I/O ports. The Matlab/Simulink package Real-time Workshop allows the computer to directly interface with the DAQcards.

During the initial development of the control laws it was discovered that poor signal quality from the sensors was significantly degrading the controllability of the TASS. In order to achieve adequate performance, signal processing was required in order to extract the signal data.

\section{$\underline{\text { Rate Gyros Signal Processing }}$}

The signal generated by the rate gyros has two characteristics that make it difficult to utilize. First, the gyro is noisy, and this noise is significant at the near-zero rates encountered during normal operation. Second, the gyro experiences a bias that slowly varies during operation, and varies widely day-to-day (i.e. between on-off-on cycles). Several low-pass filters were considered to remove the highfrequency noise in the gyro signal. The noise was eliminated using a low-pass Butterworth filter ${ }^{4}$. This filer was chosen for its simplicity and minimal time delay imposed upon the signal. Figure 10 shows the 
rate gyro signal with the filtered signal overlaid on the raw signal.

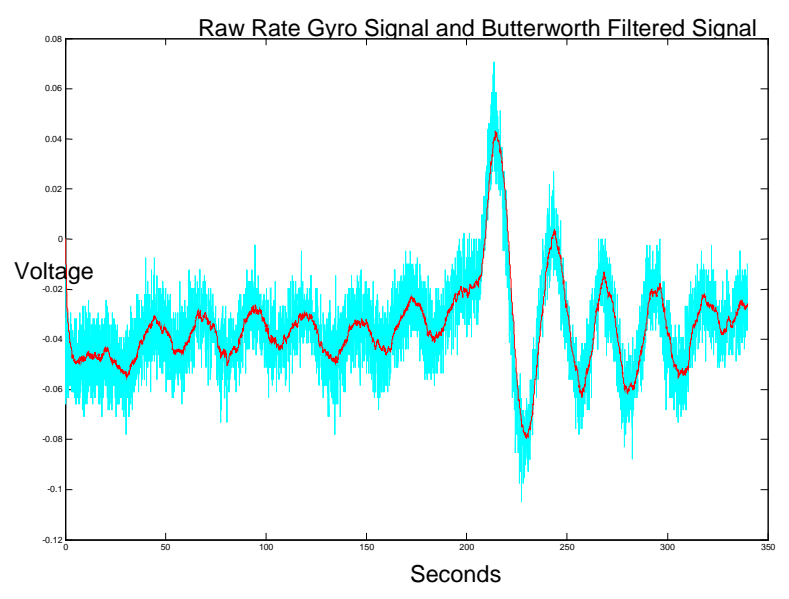

Figure 10. Raw Rate gyro Signal and $1^{\text {st }}$ Order Butterworth Filter Output

The variable bias was eliminated using a digital filter suggested by Professor Roberto Cristi. The filter is characterized by the equations:

$$
\begin{gathered}
y(n)=a y(n-1)+b[x(n)-x(n-1)] \\
0<a<1 \\
b=\frac{1+a}{2}
\end{gathered}
$$

This digital filter is based on the assumption that the rate will always oscillate about zero. It will produce erroneous results if used while slewing the TASS through large angles, or if there is a significant balancing bias on the table with 'loose' position gains. The combination of the Butterworth filter and the above digital filter is referred to as the 'CC Filter' ('CC' refers to 'Cristi-Chernesky').

A value of $a=0.99$ was chosen to minimize the data (amplitude) loss resulting from the digital filter. The consequence of this robust response is a 15-20 second time period required for the filter to initially zero out the gyro bias, as shown in Figure 11. As the value of $a$ gets larger less of the signal (vice noise) is filtered out, but at the cost of a longer time response.

Sun Sensor Signal ProcessingAs discussed in the hardware section, the sun sensor has a stable, linear response for any given position of the sun/star constellation. However, the signals from the sun sensor also have a quantization, or graininess, in their response.

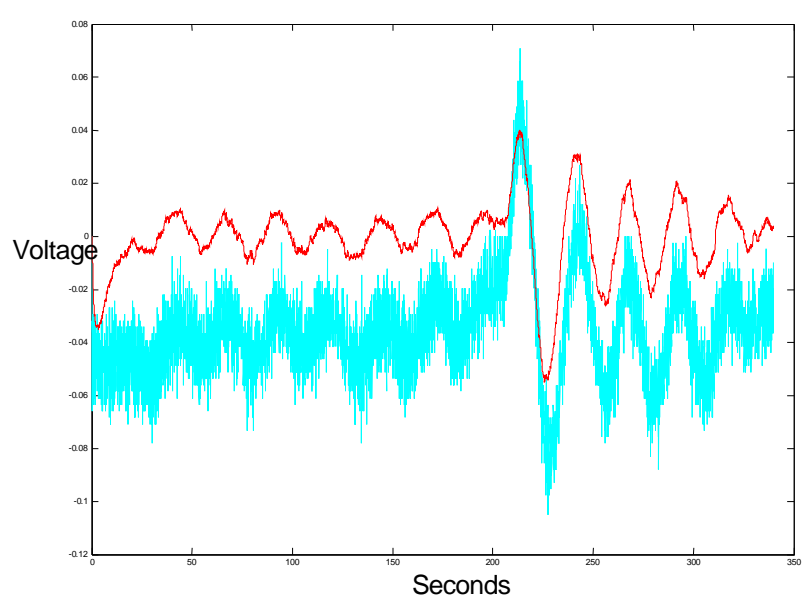

Figure 11. Raw Rate Gyro Signal and CC Filter Output

After testing several filter designs, a Butterworth filter ${ }^{4}$ was chosen to smooth the sun sensor data. Care was taken in selecting the order of the filter, as higher order filters had a significant time delay associated with them. It was experimentally determined that a time delay of greater than roughly 0.5 seconds produced unacceptable controllability given a slight off-balance table condition. A firstorder filter was chosen based on this data.

\section{Controller Development}

The initial controller provided with the TASS at delivery was optimized for thruster control, utilizing a Proportional-Derivative (PD) controller. This provided acceptable attitude control with the higher-torque thruster system, but marginal performance when using only the reaction wheel system. This PD system was used as a basis for the initial reaction wheel only controller.

The first of these refinements was the incorporation of integral control to the control law. This was a result of the difficulties in fine balancing the table. The table was extremely difficult to 'perfectly' balance, and the PD controller allowed the TASS to stabilize away from the intended commanded position (A function of the constant, imbalance-related bias). This large position error was inconsistent with the accuracy required for laser alignment with the primary mirror. The PID controller eliminates this large steady-state error due to slight table imbalance.

Another refinement was a result of the principal axis offset. The control axes are offset from the principal axes by 45 degrees. All input signals (sun sensor and rate gyros) were shifted from the control axis system to the principal axis system by multiplying by a direction cosine matrix (DCM). 
These signals were then used in the PID controller, and the outputs passed through the inverse DCM to shift the output signal back into the control coordinate frame. These output signals are then sent to the reaction wheels. This allows for independent control around each principal axis, while retaining the hardware configuration on the table. This also allows for future modifications to the TASS that may alter the location of the principal axes.

The final issue in controller development was the time delay associated with the $\mathrm{CC}$ filter. Roughly 20 seconds is required for the current filter to zero out the variable bias in the rate gyros. A time delay is implemented which prevents control output to the reaction wheels for the specified time, and blocks any accumulation of integral control signal. Operationally, the TASS is grounded during these initial 20 seconds, ensuring the rates are drawn to zero bias at the beginning of operation.

\section{RESULTS}

The TASS was well balanced for test, and the batteries were in a state of full charge. All systems had been tested the day prior, and the TASS sun sensor position was from the previous days operations. The gains used for this test were as follows, (PID: X 0.5/0.05/0.001; Y 0.4/0.03/0; Z 0.7/0.04/0.001).

The position of the table as measured by the sun sensors is shown in Figure 12. The scales on all three axes is from $-3^{\circ}$ to $3^{\circ}$, clearly showing the quantization in each axis. The red lines indicate the commanded position. The $\mathrm{X}_{\mathrm{C}}$ and $\mathrm{Z}_{\mathrm{C}}$ axes were very well controlled around the commanded position, however the $\mathrm{Y}_{\mathrm{C}}$ axis was not. The table drifted a significant distance away from the commanded position before correcting. This loose control was
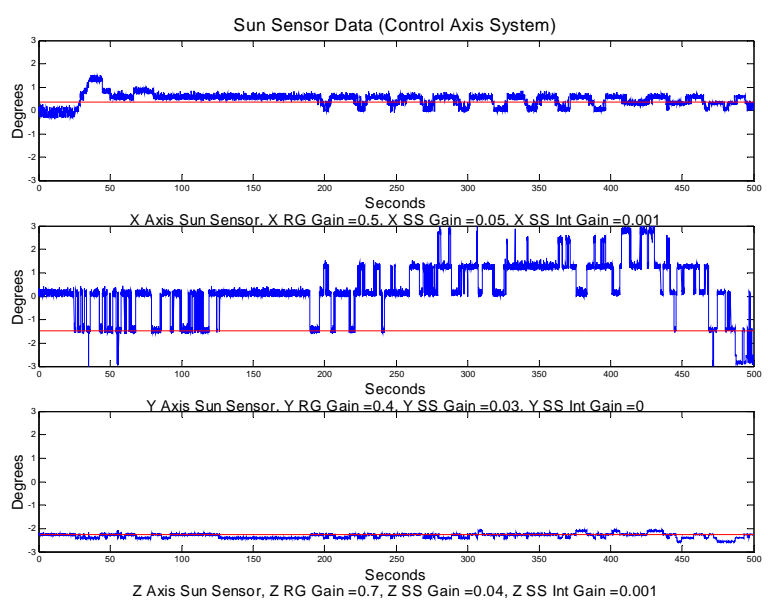

Figure 12. TASS Position Data required due to the poor sensor quality. If higher position gains were used, the TASS fluctuated around the commanded position. This axis was also the axis of the highest moment of inertia, and thus the reaction wheel had the least control authority.

Figure 13 is the filtered data being fed into the control law from the sun sensors. This data had been through a DCM to align it with the principal axes, and filtered using a first order Butterworth filter. The vertical axes here are in volts, vice degrees. This figure illustrates that although the first order Butterworth filter helps smooth the $\mathrm{Y}_{\mathrm{P}}$-axis data, the signal is still very jagged.
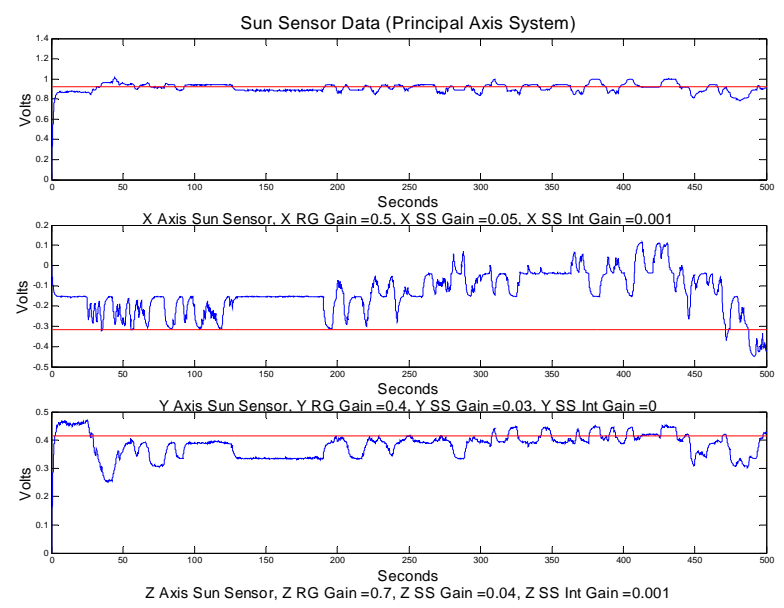

Figure 13. Filtered Sun Sensor Data in Principal Axes

Figure 14 shows the raw rate gyro signal in the control axis frame, and Figure 15 shows the filtered signals in the principal axis frame. The improvement in signal quality is obvious. The spike in the raw data at time 20 s is due to the TASS lifting off the air bearing. The flat portion for the first 20 seconds is the time delay in the SIMULINK model blocking any control output during the time delay period. The laser testing proceeded normally from time 20 s to 250 s, when the TASS drifted out of tolerance in the $\mathrm{Y}_{\mathrm{C}}$ axis. At this time the bench-top laser was no longer striking the primary mirror, and did not re-acquire until time 480s. At time 480s the table had a large positive rate, and acquisition lasted only a few seconds before the table went out of tolerance again. The period between 20 s and 250 s allowed sufficient time to test the optical train in all modes. Target tracking was accomplished, with the red laser successfully tracking the green laser. The 'Draw Square' and 'Draw Expanding Circle' features were also tested, indicating proper function of the FSM and associated electronics. 

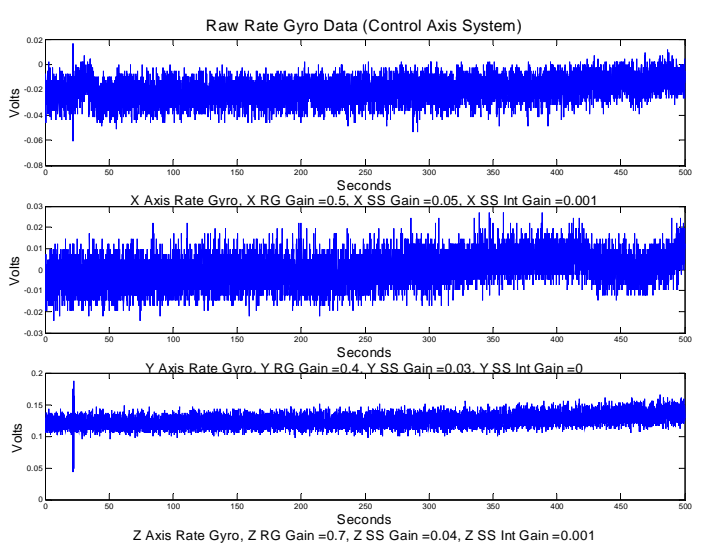

Figure 14. Raw Rate Gyro Data, Control Axis System
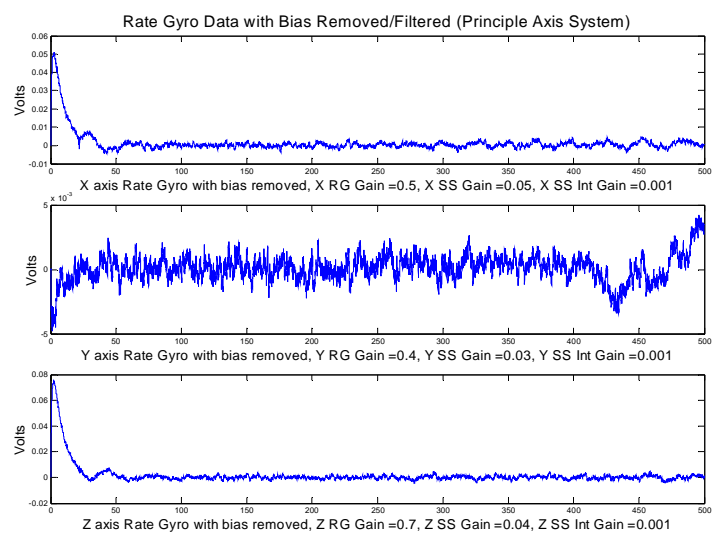

Figure 15. Filtered Rate Gyro Data, Principal Axis System

\section{$\underline{\text { SUMMARY AND CONCLUSIONS }}$}

This experimentation and development with the TASS and BRMP has demonstrated technologies vital to the Bifocal Relay Mirror project. Beam steering, control, and jitter reduction within the BRMP were successful, and system performance of the TASS was successful, with the exception of sun sensor position accuracy about one axis.
The TASS hardware saw many changes, including the integration of the BRMP. The mass properties have been analytically determined and validated against experimental data. Signal processing algorithms were applied to the rate gyro and sun sensor signals, extracting useful data in all cases except the $\mathrm{Y}_{\mathrm{C}}$-axis sun sensor.. The $\mathrm{Y}_{\mathrm{C}}$-axis sun sensor was determined to have insufficient resolution for sustained BRMP operations, and alternative attitude determination methods were explored. The PID controller implemented was successful, as were the direction cosine matrices used to shuttle between control axes and principal axes.

The TASS is a test bed for satellite attitude control research in support of experimental payloads such as the bifocal relay mirror. It has evolved throughout this experiment, and it will continue to evolve as future experiments present new requirements. As a student tool, and as hardware supporting national interests, the Three Axis Satellite Simulator continues to be a successful program within the Spacecraft Research and Design Center at NPS.

\section{ACKNOWLEDGMENTS}

The authors would like to thank Prof. Brij N. Agrawal, Director of the Spacecraft Research and Design Center at Naval Postgraduate School, and Dr. William Harter of the National Reconnaissance Office and the Directors Innovation Initiative.

\section{REFERENCES}

1. Strategic Defense Initiative Organization, Program Summary: Relay Mirror Experiment and Wideband Angular Vibration Experiment, Washington, D.C., 1992.

\section{Advanced Spacecraft Design Team, MITHRA} Relay Mirror Satellite, Naval Postgraduate School, Monterey, California, September 2000.

3. National Reconnaissance Office (AS\&T), Director's Innovation Initiative: FY 2001 Government Sources Sought Announcement, Chantilly, Virginia, 2001.

4. Strum, R. D. and Kirk, D.E., Discrete Systems and Digital Signal Processing, Addison-Wesley, 1989. 